\title{
Impact of aging on cardiac function in a female rat model of menopause: role of autonomic control, inflammation, and oxidative stress
}

\author{
Jacqueline Freire Machi, ${ }^{1,2}$ \\ Danielle da Silva Dias ${ }^{3}$ \\ Sarah Cristina Freitas ${ }^{3}$ \\ Oscar Albuquerque de \\ Moraes' \\ Maikon Barbosa da Silva' \\ Paula Lázara Cruz' \\ Cristiano Mostarda ${ }^{4}$ \\ Vera M C Salemi' \\ Mariana Morris ${ }^{2}$ \\ Kátia De Angelis ${ }^{3}$ \\ Maria-Cláudia Irigoyen' \\ 'Hypertension Unit, Heart Institute \\ (InCor), School of Medicine, \\ University of Sao Paulo, São Paulo, \\ Brazil; '2Institute of Neuro-Immune \\ Medicine, Nova Southeastern \\ University, Fort Lauderdale, FL, \\ USA; ${ }^{3}$ Laboratory of Translational \\ Physiology, Universidade Nove de \\ Julho (UNINOVE), São Paulo, ${ }^{4} \mathrm{Health}$ \\ Adult and Child, Federal University \\ of Maranhao (UFMA), São Luiz, \\ Maranhão, Brazil
}

\author{
This article was published in the following Dove Press journal: \\ Clinical Interventions in Aging \\ 22 March 2016 \\ Number of times this article has been viewed
}

\begin{abstract}
Objective: The aim of this study was to evaluate the effects of aging on metabolic, cardiovascular, autonomic, inflammatory, and oxidative stress parameters after ovarian hormone deprivation (OVX).
\end{abstract}

Methods: Female Wistar rats ( 3 or 22 months old) were divided into: young controls, young ovariectomized, old controls, and old ovariectomized (bilateral ovaries removal). After a 9-week follow-up, physical capacity, metabolic parameters, and morphometric and cardiac functions were assessed. Subsequently, arterial pressure was recorded and cardiac autonomic control was evaluated. Oxidative stress was measured on the cardiac tissue, while inflammatory profile was assessed in the plasma.

Results: Aging or OVX caused an increase in body and fat weight and triglyceride concentration and a decrease in both insulin sensitivity and aerobic exercise capacity. Left ventricular diastolic dysfunction and increased cardiac overload (myocardial performance index) were reported in old groups when compared with young groups. Aging and OVX led to an increased sympathetic tonus, and vagal tonus was lower only for the old groups. Tumor necrosis factor- $\alpha$ and interleukin-6 were increased in old groups when compared with young groups. Glutathione redox balance (GSH/GSSG) was reduced in young ovariectomized, old controls, and old ovariectomized groups when compared with young controls, indicating an increased oxidative stress. A negative correlation was found between GSH/GSSG and tumor necrosis factor- $\alpha$ $(r=-0.6, P<0.003)$. Correlations were found between interleukin- 6 with adipose tissue $(r=0.5$, $P<0.009)$ and vagal tonus $(r=-0.7, P<0.0002)$; and among myocardial performance index with interleukin-6 ( $r=0.65, P<0.0002)$, sympathetic tonus $(r=0.55, P<0.006)$, and physical capacity $(r=-0.55, P<0.003)$. The findings in this trial showed that ovariectomy aggravated the impairment of cardiac and functional effects of aging in female rats, probably associated with exacerbated autonomic dysfunction, inflammation, and oxidative stress.

Keywords: autonomic nervous system, aging, aerobic exercise, female rats

\section{Introduction}

The aging process or senescence is related to morphological and functional changes in the cardiovascular system, with great emphasis on incidence of cardiovascular disease (CVD). ${ }^{1}$ CVD is the leading cause of morbidity and mortality in the world. In 2012, an estimated 17.5 million people died from CVDs, representing $31 \%$ of global deaths. ${ }^{2}$ In addition, CVD develops later in women than in men and is still the major cause of death in women. It is also estimated that 43 million women in the US are affected by CVDs, which strengthens the hypothesis that age and sex play a significant role in the prevalence of cardiovascular risk. ${ }^{3,4}$ This hypothesis postulates that climacteric women are exposed to increased cardiovascular risk, since aging and menopause have been (c)
hereby accept the Terms. Non-commercial uses of the work are permitted without any further permission from Dove Medical Press Limited, provided the work is properly attributed. For permission

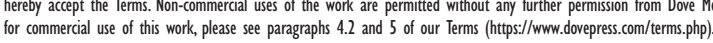


associated with decreased exercise capacity, muscle strength, and bone mass, but increased body weight and high diabetes prevalence. In addition, the climacteric period worsens not only hemodynamic profile, ${ }^{5,6}$ but also the autonomic balance decreasing parasympathetic and/or increasing sympathetic activity with associated changes in cardiac function, inflammatory and oxidative stress markers.

Therefore, this experimental trial was designed to evaluate the hypothesis that early ovarian hormone deprivation (OVX) and/or aging may lead to sympathetic activation, which may interact with inflammation and oxidative stress, causing cardiac dysfunction and physical capacity loss, as observed in postmenopausal young or older women. To test this hypothesis, the effects of aging were assessed on metabolic, cardiovascular, autonomic, inflammatory, and oxidative stress parameters after OVX.

\section{Methods}

Experiments were performed on 32 female rats. 163 -monthold and 16 22-month-old rats (Rattus norvegicus) obtained from the Federal University of São Paulo (UNIFESP) were maintained in the animal house facility of the Heart Institute of the University of Sao Paulo, receiving standard laboratory chow and water ad libitum. The animals were housed in individual cages in a temperature-controlled room $\left(22^{\circ} \mathrm{C}-24^{\circ} \mathrm{C}\right)$ with a 12-hour dark-light cycle.

This study was approved by the Experimental Animal Use Committee of the School of Medicine, University of São Paulo (protocol 360/11), and were conducted in accordance with the National Institute of Health Guide for the Care and Use of Laboratory Animals.

The rats were assigned to four groups: young controls ( $\mathrm{YC}=8$ ), young ovariectomized ( $\mathrm{YO}=8$ ), old controls $(\mathrm{OC}=8)$, and old ovariectomized $(\mathrm{OO}=8)$. In order to avoid any influence of the fluctuation of female sex hormones on the results observed in the YC and OC groups, the identification of the stage of the estrous cycle of the rats was performed. All experimental evaluations were performed in nonovulatory phases of the estrous cycle of rats. ${ }^{7}$

\section{Ovariectomy}

After 3 months (YO) and 22 months (OO) of life, the animals were anesthetized $(80 \mathrm{mg} / \mathrm{kg}$ ketamine and $12 \mathrm{mg} / \mathrm{kg}$ xylazine, intraperitoneal [IP]), and a small abdominal incision was performed. The oviduct was sectioned and the ovary removed as described in detail elsewhere. ${ }^{8,9}$

Estrogen concentration in the blood was measured by immunoassay to confirm OVX. ${ }^{10}$

\section{Physical capacity}

All animals were adapted (1 week after ovariectomy) to the treadmill (TK-01; Ibramed, Porto Alegre, Brazil) (10 min/day; $0.3 \mathrm{~km} / \mathrm{h}$ ) for 1 week prior to the maximal treadmill test. A maximal treadmill test ${ }^{11}$ was performed by all groups: at the beginning of the experiment and in the 4th and 8th weeks of the protocol. The purpose was to determine exercise capacity.

\section{Determination of blood glucose and triglycerides}

At the end of the protocol, all animals underwent 4 hours of fasting. A drop of blood from the tail was collected to measure plasma glucose using a glucometer, and another drop was collected for triglyceride measurement with Accutrend GTC (Hoffman-La Roche Ltd., Basel, Switzerland).

\section{Insulin resistance test}

At the end of the protocol, all animals underwent a 2-hour fasting and were anesthetized with sodium pentobarbital (40 mg/kg IP). Plasma glucose was measured from blood samples collected from the animal cannula with the use of a glucometer (Accu-Check; Hoffman-La Roche Ltd.) at times $0,4,8,12$, and 16 minutes after an insulin intravenous injection $(0.75 \mathrm{U} / \mathrm{kg}$ body weight). Glycemia values for 4-16 minutes were used to calculate the constant decrease of plasma glucose. ${ }^{12}$

\section{Echocardiography}

At the end of the protocol, a transthoracic echocardiography was performed in all the groups using double-blind observers in accordance with the guidelines of the American Society of Echocardiography. Rats were anesthetized $(50 \mathrm{mg} / \mathrm{kg}$ ketamine and $12 \mathrm{mg} / \mathrm{kg}$ xylazine, IP) and the echocardiographic parameters were measured as previously described. ${ }^{13,14}$

\section{Cardiovascular measurements}

Ten weeks after the beginning of protocol, two catheters filled with saline solution were implanted in anesthetized rats $(80 \mathrm{mg} / \mathrm{kg}$ ketamine and $12 \mathrm{mg} / \mathrm{kg}$ xylazine, IP), into the artery and femoral vein (PE-50) for direct measurements of arterial pressure (AP) and drug administration, respectively. Twentyfour hours after surgical procedures, the arterial cannula was connected to a transducer (Kent Instruments, Kent Scientific Corporation, Torrington, Connecticut, USA), and AP signals were recorded over a 30 -minute period by a microcomputer equipped with an analog-to-digital converter board Windaq (DATAQ Instruments, Akron, OH, USA). Rats were conscious and allowed to move freely during the experiments. Increasing 
doses of phenylephrine $(0.25-32 \mu \mathrm{g} / \mathrm{kg})$ and sodium nitroprusside $(2.5$ a $100 \mu \mathrm{g} / \mathrm{kg})$ were given as sequential bolus injections $(0.1 \mathrm{~mL})$ to produce pressure responses ranging from 5 to $40 \mathrm{mmHg}$. A 3- to 5-minute interval between doses was necessary for blood pressure to return to baseline. Peak increases or decreases in mean AP (MAP) after phenylephrine or sodium nitroprusside injection and the corresponding peak reflex changes in heart rate (HR) were recorded for each dose of the drug. Baroreceptor reflex sensitivity was evaluated by a mean index relating changes in HR to changes in MAP, allowing a separate analysis of gain for reflex bradycardia and reflex tachycardia. The mean index was expressed as beats per minute per millimeter of mercury, as described elsewhere. ${ }^{9}$

Vagal and the sympathetic tonus and effects and intrinsic heart rate (IHR) were measured by determining the response to methylatropine (3 $\mathrm{mg} / \mathrm{kg}$ intravenous [IV]) and propranolol (4 mg/kg IV) after basal AP recording, as described in detail elsewhere. ${ }^{9}$ AP and HR were continuously recorded at a basal state and after methylatropine ( $3 \mathrm{mg} / \mathrm{kg}$ IV) injection $(<0.2 \mathrm{~mL})$. Because the HR response to this drug reaches its peak within 3-5 minutes, this time interval was allowed to elapse before HR measurement. Propranolol (4 mg/kg IV) was injected $(<0.2 \mathrm{~mL}) 10$ minutes after methylatropine injection, and once again, the response was evaluated after simultaneous blockade with propranolol and methylatropine. On the subsequent day, the sequence of injections was inverted (first propranolol and then methylatropine). The sympathetic effect was determined as the difference between basal HR and the lower HR after propranolol injection, vagal effect was obtained by the difference between maximum HR after methyl atropine injection and basal HR, ultimately sympathetic and vagal tonus were evaluated by intrinsic HR instead of HR. ${ }^{6,15}$

\section{Body weight and visceral white adipose tissue}

Animals were weighed once a week during 10 weeks of protocol. The visceral white adipose tissue (WAT) was collected and weighed at the end of protocol on an analytical balance for comparison among groups.

\section{Inflammatory markers on plasma}

Interleukin (IL) 1B, IL-4, IL-6, and tumor necrosis factor (TNF)- $\alpha$ levels in plasma were determined using a commercially available enzyme-linked immunosorbent assay kit (R\&D Systems Inc., Minneapolis, MN, USA), in accordance with the manufacturer's instructions. Enzyme-linked immunosorbent assay was performed in 96-well polystyrene microplates using specific monoclonal antibody coating.

\section{Oxidative stress profile}

One day after hemodynamic evaluations, the animals were euthanized and the heart (left ventricles) was immediately removed, rinsed in saline, and trimmed to remove fat tissue and visible connective tissue. The tissue was cut into small pieces, placed in ice-cold buffer, and homogenized in an Ultra80 Turrax blender (UltraStirrer, Pilatusstrasse, Muri, Switzerland) with $1 \mathrm{~g}$ tissue per $4 \mathrm{~mL} 120 \mathrm{mM} \mathrm{KCl}$ and $20 \mathrm{nM}$ sodium phosphate buffer, $\mathrm{pH}$ 7.4. The homogenate was centrifuged at $600 \times g$ for 10 minutes at $-26^{\circ} \mathrm{C}$.

\section{Lipoperoxidation by thiobarbituric acid reactive substances}

For the thiobarbituric acid reactive substance (TBARS) assay, trichloroacetic acid $(10 \%, \mathrm{w} / \mathrm{v})$ was added to the homogenate to precipitate proteins and to acidify the samples. This mixture was then centrifuged $(1,000 \times g, 3$ minutes $)$, the protein-free sample was extracted, and thiobarbituric acid $(0.67 \%, \mathrm{w} / \mathrm{v})$ was added to the reaction medium. The tubes were placed in a water bath $\left(100^{\circ} \mathrm{C}\right)$ for 15 minutes Absorbance was measured at $535 \mathrm{~nm} .{ }^{16}$

\section{Protein oxidation by carbonyl assay}

Tissue samples were incubated with2,4-dinitrophenylhydrazine $(10 \mathrm{mM})$ in a $2.5 \mathrm{M} \mathrm{HCl}$ solution for 1 hour in the dark. Samples were vortexed every 15 minutes. Subsequently, a $20 \%$ trichloroacetic acid (w/v) solution was added and the solution was incubated on ice for 10 minutes and centrifuged for 5 minutes at $1,000 \times g$ to collect protein precipitates. An additional wash was performed with $10 \%$ trichloroacetic acid $(\mathrm{w} / \mathrm{v})$. The pellet was washed three times with ethanolethyl acetate $(1: 1)(\mathrm{v} / \mathrm{v})$. The final precipitates were dissolved in $6 \mathrm{M}$ guanidine hydrochloride solution and incubated for 10 minutes at $37^{\circ} \mathrm{C}$, and absorbance was measured at $360 \mathrm{~nm} \cdot{ }^{17}$

\section{Antioxidant enzyme activities}

The quantification of superoxide dismutase activity was based on the inhibition of the reaction between $\mathrm{O}_{2} \cdot{ }^{-}$and pyrogallol. ${ }^{18}$ Catalase activity (CAT) was determined by measuring the decrease in $\mathrm{H}_{2} \mathrm{O}_{2}$ absorbance at $240 \mathrm{~nm} .{ }^{19}$ Glutathione peroxidase (GPx) activity was based on the consumption of NADPH at $480 \mathrm{~nm} \cdot{ }^{20}$

\section{Glutathione redox balance}

To determine oxidized and reduced glutathione concentration, tissue was deproteinized with $2 \mathrm{~mol} / \mathrm{L}$ perchloric acid, centrifuged during 10 minutes at $1,000 \times \mathrm{g}$, and 
the supernatant was neutralized with $2 \mathrm{~mol} / \mathrm{L}$ potassium hydroxide. The reaction medium contained $100 \mathrm{mmol} / \mathrm{L}$ phosphate buffer ( $\mathrm{pH} 7.2$ ), $2 \mathrm{mmol} / \mathrm{L}$ nicotinamide dinucleotide phosphate acid, $0.2 \mathrm{U} / \mathrm{mL}$ glutathione reductase, and

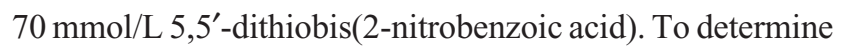
reduced glutathione, the supernatant was neutralized with $2 \mathrm{~mol} / \mathrm{L}$ potassium hydroxide, to react with $70 \mathrm{mmol} / \mathrm{L} \mathrm{5,5^{ \prime } -}$ dithiobis(2-nitrobenzoic acid), and the absorbance values measured at $420 \mathrm{~nm}^{21}$

\section{Statistical analysis}

Data were reported as mean \pm standard error of the mean, and (one- or two-way) analysis of variance followed by Student's $t$-test and Newman-Keuls post hoc test was used to compare the groups. Associations between variables were analyzed by Pearson's correlation. Differences were considered significant when $P<0.05$.

\section{Results \\ Metabolic evaluations}

The initial body weight was higher in OO and OC groups when compared with young groups ( $\mathrm{YC}$ and $\mathrm{YO}$ ). At the end of the protocol, both the YO and YC groups showed a significant increase in body weight gain when compared with the initial week, while the OC and OO groups did not. The ovariectomy has caused an additional increase in body weight in YO group when compared with the YC (Table 1).

$\mathrm{OC}$ and $\mathrm{OO}$ groups presented a significant increase of triglyceride levels when compared with the $\mathrm{YC}$ and $\mathrm{YO}$ groups (Table 1). No differences between groups have been observed regarding blood glucose and cholesterol (Table 1).

The constant decrease of plasma glucose was lower in the OC and OO groups at the end of the protocol when compared with the YC. No differences have been shown between $\mathrm{OO}$ and $\mathrm{YO}$ groups in this parameter (Table 1), which is an indicator of insulin-resistant state induced by age or OVX.

The WAT weight at the end of the protocol was increased in the $\mathrm{YO}, \mathrm{OC}$, and $\mathrm{OO}$ groups when compared with $\mathrm{YC}$ group (Table 1).

\section{Physical capacity}

Regarding the speed in the maximum physical capacity test, both ovariectomized groups (YO and OO) have shown reduced capacity when compared with both $\mathrm{YC}$ and $\mathrm{OC}$ groups at the end of the study, suggesting that ovariectomy has caused a further loss of this capacity, not only in the old animals, but also in the young ones. The OO group presented an additional impairment in the physical capacity when compared with OC group (Table 1).

\section{Cardiac morphometry}

Echocardiographic evaluation data, as shown in Table 2, have shown that left ventricular mass and left ventricular diameter during diastole increased in older groups (OC and $\mathrm{OO}$ ) when compared with young groups ( $\mathrm{YC}$ and $\mathrm{YO}$ ). No differences were observed between groups regarding relative wall thickness (Table 2).

\section{Cardiac function}

In young animals, there were no significant differences in systolic and diastolic function. Moreover, no differences were observed regarding ejection fraction and shortening fraction among all groups. As to diastolic function, the $\mathrm{E} / \mathrm{A}$ ratio and isovolumetric relaxation time observed in the $\mathrm{OC}$ and $\mathrm{OO}$ groups were increased when compared with the $\mathrm{YC}$ and $\mathrm{YO}$ groups. E wave deceleration time (Dec E) was impaired only in the OO group when compared with YO. On the other hand, the myocardial performance index (MPI), which is the ratio of total time spent in isovolumic activity (isovolumic

Table I Metabolic evaluations and physical capacity in YC, YO, OC, and OO groups

\begin{tabular}{|c|c|c|c|c|c|}
\hline Parameters/groups & YC & YO & OC & O० & $P$-value \\
\hline \multicolumn{6}{|l|}{ Body weight (g) } \\
\hline Initial & $202.0 \pm 6.04$ & $227.4 \pm 5.02$ & $250.9 \pm 10.13^{*, \#}$ & $270.0 \pm 11.07 * \#$ & 0.0001 \\
\hline Final & $220.6 \pm 6.59^{\ddagger}$ & $263.3 \pm 5.56^{*, \neq}$ & $251.4 \pm 12.78$ & $271.0 \pm 11.38 *$ & 0.0001 \\
\hline Adipose tissue $(\mathrm{g})$ & $3.78 \pm 0.51$ & $6.55 \pm 0.88^{*}$ & $6.56 \pm 0.65^{*}$ & $6.27 \pm 0.65^{*}$ & 0.001 \\
\hline Triglycerides (mg/dL) & $109.85 \pm 5.90$ & $113.50 \pm 5.68$ & $124.55 \pm 8.95^{*, \#}$ & $|30.80 \pm 5.8|^{*, \#}$ & 0.05 \\
\hline Glycemia (mg/dL) & $88.13 \pm 1.14$ & $84.75 \pm 0.79$ & $83.40 \pm 5.06$ & $84.20 \pm 7.70$ & 0.331 \\
\hline Cholesterol (mg/dL) & $166.13 \pm 1.44$ & $164.13 \pm 1.23$ & $169.00 \pm 3.64$ & $164.29 \pm 1.55$ & 0.389 \\
\hline kITT (\%/min) & $4.4 I \pm 0.36$ & $3.63 \pm 0.22$ & $3.33 \pm 0.17 *$ & $3.18 \pm\left. 0.3\right|^{*}$ & 0.001 \\
\hline Physical capacity $(\mathrm{km} / \mathrm{h})$ & $1.98 \pm 0.07$ & $1.67 \pm 0.08 *$ & $1.35 \pm 0.05^{*, \#}$ & $1.02 \pm 0.05^{*, \#, *}$ & 0.0001 \\
\hline
\end{tabular}

Notes: Data are represented as mean \pm standard error of the mean. $* P<0.05$ vs $Y C,{ }^{\sharp} P<0.05$ vs OC, ${ }^{\sharp}<0.05$ vs $Y O$, ${ }^{\ddagger} P<0.05$ vs $O O$.

Abbreviations: kITT, constant decrease of plasma glucose; OC, old (24 months old) control; OO, old ovariectomized; YC, young (5 months old) control; YO, young ovariectomized. 
Table 2 Echocardiographic measurements in YC, YO, OC, and OO groups

\begin{tabular}{|c|c|c|c|c|c|}
\hline Parameters/groups & YC & YO & OC & OO & $P$-value \\
\hline \multicolumn{6}{|l|}{ Morphometry } \\
\hline $\operatorname{LVM}(\mathrm{g} / \mathrm{kg})$ & $1.01 \pm 0.08$ & $1.01 \pm 0.02$ & I. $12 \pm 0.03^{*, \#}$ & $1.12 \pm 0.04^{* \#}$ & 0.004 \\
\hline RWT & $0.62 \pm 0.04$ & $0.63 \pm 0.03$ & $0.56 \pm 0.05$ & $0.61 \pm 0.03$ & 0.443 \\
\hline LVDIA (cm) & $0.51 \pm 0.009$ & $0.56 \pm 0.02$ & $0.65 \pm 0.03^{\text {*\# }}$ & $0.61 \pm 0.02 *$ & 0.004 \\
\hline \multicolumn{6}{|l|}{ Systolic function } \\
\hline $\mathrm{EF}(\%)$ & $80.12 \pm 3.05$ & $80.75 \pm 2.85$ & $85.44 \pm 1.62$ & $87.29 \pm 3.19$ & 0.172 \\
\hline SF (\%) & $42.94 \pm 3.34$ & $43.05 \pm 2.72$ & $48.36 \pm 1.97$ & $52.26 \pm 4.22$ & 0.355 \\
\hline VCF $($ circ/s $\times 100)$ & $0.43 \pm 0.01$ & $0.44 \pm 0.03$ & $0.46 \pm 0.02$ & $0.46 \pm 0.04$ & 0.972 \\
\hline \multicolumn{6}{|l|}{ Diastolic function } \\
\hline IVRT (ms) & $28.6 \pm 0.60$ & $27.25 \pm 1.04$ & $40.69 \pm 4.49 *, \#$ & $41.40 \pm 3.14^{*, \#}$ & 0.01 \\
\hline Dec E (ms) & $57.40 \pm 3.58$ & $49.42 \pm 3.72$ & $59.22 \pm 1.32$ & $62.45 \pm 3.50^{\#}$ & 0.04 \\
\hline E/A & $2.04 \pm 0.08$ & $2.19 \pm 0.13$ & $1.74 \pm 0.08^{*, \#}$ & $1.60 \pm 0.06^{* \# \#}$ & 0.001 \\
\hline \multicolumn{6}{|l|}{ Global function } \\
\hline MPI & $0.25 \pm 0.03$ & $0.26 \pm 0.03$ & $0.43 \pm 0.05^{* \#}$ & $0.40 \pm 0.05^{* \#}$ & 0.05 \\
\hline
\end{tabular}

Notes: Data are represented as mean \pm standard error of the mean. $* P<0.05$ vs $Y C,{ }^{*} P<0.05$ vs $Y O$.

Abbreviations: Dec E, E wave deceleration time; E/A, E wave and A wave ratio; EF, ejection fraction; IVRT, isovolumetric relaxation time; LVDIA, left ventricular diameter during diastole; LVM, left ventricular mass; MPI, myocardial performance index; OC, old (24 months old) control; OO, old ovariectomized; RWT, relative wall thickness; SF, shortening fraction; VCF, velocity of circumferential fiber shortening; YC, young (5 months old) control; YO, young ovariectomized.

contraction time and isovolumic relaxation time) to the ejection time, an index of cardiac overload, was higher in OC and $\mathrm{OO}$ than in $\mathrm{YC}$ and $\mathrm{YO}$ groups (Table 2).

\section{Hemodynamic and autonomic assessments}

Ovariectomy induced an increase in MAP values in $\mathrm{YO}$ and OO groups when compared with the YC and OC groups (YC, $107 \pm 1.47$; YO, $117 \pm 2.04$; OC, $110 \pm 1.67$; OO, $119 \pm 1.86 \mathrm{mmHg}$, $P<0.001)$. OVX, when associated with aging process, did not show any additional increase than already observed in $\mathrm{YO}$ to MAP. HR was increased in OC and $\mathrm{OO}$ at the end of the protocol when compared with $\mathrm{YC}$ and $\mathrm{YO}$ groups (YC, 354 \pm ;

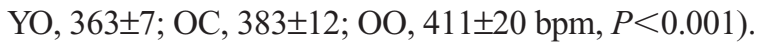

The baroreceptor reflex sensitivity evaluation has shown that the baroreflex tachycardic responses were reduced in $\mathrm{YO}, \mathrm{OC}$, and $\mathrm{OO}$ rats in relation to controls at rest. Additionally, OO had fewer responses when compared with YO. The baroreflex bradycardic responses were significantly reduced in old animals as compared with controls at rest (Table 3).

Aging has caused reduced vagal tonus. Indeed, the OC and $\mathrm{OO}$ groups had lower vagal tonus in relation to $\mathrm{YC}$ and YO groups. On the other hand, ovariectomy and/or aging seem to have caused an increase in sympathetic tonus in groups $\mathrm{YO}, \mathrm{OC}$, and $\mathrm{OO}$ when compared with YC group. The aging process has reduced the vagal effect on $\mathrm{OC}$ and OO groups when compared with the young groups ( $\mathrm{YC}$ and YO) and OO has shown an additional reduction in relation to OC. In turn, sympathetic effect was higher in OC and $\mathrm{OO}$ groups when compared with $\mathrm{YC}$ and $\mathrm{YO}$ groups and increased in the $\mathrm{YO}$ rats when compared with the $\mathrm{YC}$ rats. The old groups (OC and $\mathrm{OO}$ ) have shown reduction in IHR (Table 3).

\section{Inflammatory profile}

No difference was observed in IL-4 and IL1B among all groups. However, OC and OO groups showed a significant

Table 3 Cardiac autonomic control in YC, YO, OC, and OO groups

\begin{tabular}{|c|c|c|c|c|c|}
\hline Parameters/groups & YC & YO & OC & OO & $P$-value \\
\hline Vagal tonus (bpm) & $50.57 \pm 3.33$ & $65.63 \pm 7.50$ & $|8.60 \pm 4.7| *, \#$ & $17.14 \pm\left. 4.2\right|^{*, \#}$ & 0.0001 \\
\hline Sympathetic tonus (bpm) & $40.78 \pm 10.05$ & $80.75 \pm 5.64 *$ & $82.10 \pm 6.77^{*}$ & $95.16 \pm 12.83^{*}$ & 0.004 \\
\hline Vagal effect (bpm) & $87.29 \pm 8.92$ & $80.80 \pm 8.36$ & $34.39 \pm 5.77^{* . \#}$ & $|4.38 \pm| .86^{*, \#, \#}$ & 0.0001 \\
\hline Sympathetic effect (bpm) & $23.88 \pm 4.87$ & $53.13 \pm 9.92 *$ & $92.26 \pm 7.40 * \#$ & $83.69 \pm 7.4 I^{*, \#}$ & 0.0001 \\
\hline Intrinsic HR (bpm) & $397 \pm 6.00$ & $379 \pm 5.09$ & $304 \pm 7.82 * \#$ & $3 I I \pm 9.83 * \#$ & 0.0001 \\
\hline TR (bpm/mmHg) & $4.13 \pm 0.24$ & $3.38 \pm 0.27 *$ & $2.74 \pm 0.17^{*}$ & $2.34 \pm 0.43^{* \#}$ & 0.0001 \\
\hline BR (bpm/mmHg) & $-1.47 \pm 0.08$ & $-I .5 I \pm 0.08$ & $-1.24 \pm 0.06 *, \#$ & $-0.90 \pm 0.05^{*, \#, \#}$ & 0.0001 \\
\hline
\end{tabular}

Notes: Data are represented as mean \pm standard error of the mean. ${ }^{*} P<0.05$ vs $Y C,{ }^{\# P}<0.05$ vs $Y O,{ }^{*} P<0.05$ vs $O C$.

Abbreviations: BR, bradycardic response; HR, heart rate; OC, old (24 months old) control; OO, old ovariectomized; TR, tachycardic response; YC, young (5 months old) control; YO, young ovariectomized. 
increase in IL-6 when compared with YC and YO. TNF- $\alpha$ values were increased in old controls (OC and OO) when compared with the YC group.

\section{Oxidative stress profile}

Regarding oxidative stress in cardiac tissue, protein oxidation was higher only in $\mathrm{OO}$ when compared with YC group. The membrane lipoperoxidation (TBARS) in the cardiac tissue was higher in OC and $\mathrm{OO}$ groups than in the YC group. Regarding antioxidant enzymes, no differences in superoxide dismutase activity among groups were observed. OVX increased cardiac GPx and CAT, regardless of age. Importantly, both the YO and $\mathrm{OO}$ rats showed a decreased glutathione redox balance (GSH/GSSG) when compared with YC and OC rats, thus indicating impairment in redox balance (Table 4).

\section{Correlations}

Correlations were found between increased WAT and the increase in plasma IL-6 $(r=0.5, P=0.009)$ (Figure 1A) and in cardiac sympathetic effect ( $r=0.7, P=0.0002)$, as well as in the reduction in insulin sensitivity $(r=-0.5, P=0.004)$.

Cardiac autonomic dysfunction, represented by increased sympathetic tonus (ST) $(r=0.6, P=0.002)$ and reduced vagal tonus (VT) $(r=-0.7, P=0.0002)$ (Figure 1B), was correlated with increased levels of IL-6. The levels of IL-6 were also positively correlated with levels of TNF- $\alpha(r=0.7$, $P=0.0001)$.

The TNF- $\alpha$ was inversely correlated with GSH/GSSG ( $r=-0.6, P=0.003$ ) (Figure 1C), suggesting that animals showing higher levels of inflammatory markers also presented higher levels of cardiac oxidative stress. Finally, the global cardiac function, represented by the MPI, was positively correlated with IL-6 ( $r=0.65, P=0.0002$ ) (Figure 1D), TNF- $\alpha$ $(r=0.5, P=0.008)$, and ST $(r=0.55, P=0.006)$ (Figure 1E) and negatively correlated with physical capacity $(r=-0.55$, $P=0.003$ ) (Figure 1F).

\section{Discussion}

The results in this trial are consistent with the hypothesis that early OVX and/or aging may lead to sympathetic activation, which interacts with inflammation and oxidative stress, promoting cardiac dysfunction and loss in physical capacity.

\section{Metabolic changes}

The finding of higher weight gain in young ovariectomized rats has been repeatedly described ${ }^{22}$ and has been attributed to the reduction in energy consumption caused by estrogen deprivation and higher food intake. ${ }^{23}$ In contrast, old female ovariectomized rats did not change body weight, probably due to previous weight gain, associated with the aging process. However, the accumulation of WAT - a more important predictor of CVD than total weight - was increased in all ovariectomized rats (YO, OC, and $\mathrm{OO}$ ) when compared with YC. Moreover, the increase in sympathetic effect associated with WAT weight (and possibly WAT function) may contribute to increased AP and central obesity. ${ }^{24}$ In addition, the association between WAT and plasma IL-6 increase indicates an interaction between metabolic and inflammatory system to promote deleterious changes associated with menopause.

Insulin resistance in YO group was correlated with low levels of adiponectin, ${ }^{25}$ while in old animals (OC and OO) it was associated with increased triglyceride levels. In particular, WAT has been recognized for the role that it plays in regulating energy balance, not only as a fat storage location, but also as a source of leptin and other adipokines and lipid signaling molecules, such as ceramides or diglycerides. ${ }^{26}$ Regarding metabolic parameters, only triglycerides increased in OC and OO groups, while total cholesterol was similar

Table 4 Inflammatory and oxidative stress evaluations in YC, YO, OC, and OO groups

\begin{tabular}{|c|c|c|c|c|c|}
\hline Parameters/groups & YC & YO & OC & O० & $P$-value \\
\hline IL-4 (pg/mL) & $6.57 \pm 0.33$ & $6.53 \pm 0.24$ & $7.28 \pm 0.30$ & $7.16 \pm 0.36$ & 0.234 \\
\hline$I L I B(p g / m L)$ & $59.38 \pm 6.80$ & $70.14 \pm 12.77$ & $57.58 \pm 4.90$ & $52.21 \pm 3.82$ & 0.447 \\
\hline IL-6 (pg/mL) & $224.54 \pm 5.97$ & $208.05 \pm 11.38$ & $278.09 \pm 10.57^{*, \#}$ & $253.34 \pm 7.0 I^{*, \#}$ & 0.001 \\
\hline TNF- $\alpha(p g / m L)$ & $52.87 \pm 2.30$ & $57.34 \pm 2.10$ & $68.07 \pm 3.90^{*, \#}$ & $63.12 \pm 2.36 *$ & 0.01 \\
\hline Lipoperoxidation ( $\mu \mathrm{mol} / \mathrm{mg}$ protein) & $0.93 \pm 0.09$ & $1.46 \pm 0.20^{*}$ & $1.02 \pm 0.10$ & $1.47 \pm 0.13^{*}$ & 0.01 \\
\hline Protein oxidation (nmol/mg protein) & $6.38 \pm 0.73$ & $6.73 \pm 0.5$ । & $7.37 \pm 0.35$ & $8.43 \pm 0.55 *$ & 0.05 \\
\hline CAT (nmol/mg protein) & $0.82 \pm 0.09$ & $1.48 \pm 0.13^{*}$ & $0.90 \pm 0.09^{\#}$ & $1.42 \pm 0.14^{* *} *$ & 0.003 \\
\hline SOD (USOD/mg protein) & $21.80 \pm 0.85$ & $24.33 \pm 0.86$ & $21.20 \pm 1.64$ & $23.48 \pm 0.92$ & 0.325 \\
\hline GPx $(\mu \mathrm{mol} / \mathrm{min} / \mathrm{mg}$ protein $)$ & $0.048 \pm 0.006$ & $0.072 \pm 0.008^{*}$ & $0.038 \pm 0.004^{\#}$ & $0.075 \pm 0.005^{*, *}$ & 0.0001 \\
\hline GSH/GSSG & $7.59 \pm 0.64$ & $4.25 \pm 0.25 *$ & $5.96 \pm 0.34^{* \#}$ & $4.08 \pm 0.62^{*, *}$ & 0.0001 \\
\hline
\end{tabular}

Notes: Data are represented as mean \pm standard error of the mean. $* P<0.05$ vs $Y C,{ }^{\sharp} P<0.05$ vs $Y O,{ }^{*} P<0.05$ vs $O C$.

Abbreviations: CAT, catalase activity; GPx, glutathione peroxidase; GSH/GSSG, glutathione redox balance; IL, interleukin; OC, old (24 months old) control; OO, old ovariectomized; SOD, superoxide dismutase; TNF, tumor necrosis factor; YC, young (5 months old) control; YO, young ovariectomized. 

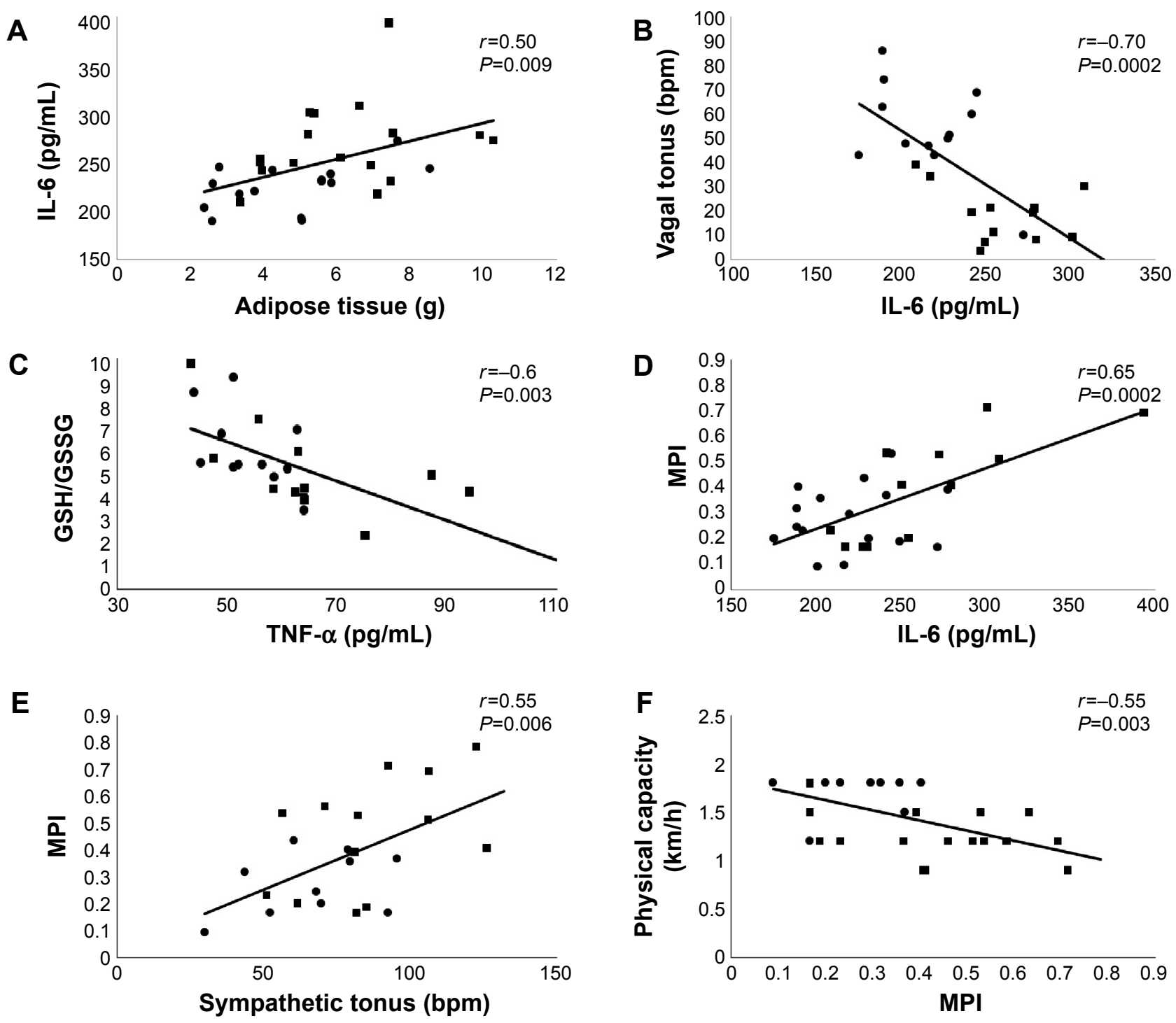

Figure I Pearson's correlation analysis involving all studied groups: $(\bullet)$ represent $Y C$ and $Y O$ rats and $(\bullet)$ represent $O C$ and $O O$ rats.

Notes: (A) Adipose tissue and IL-6 ( $r=0.5, P<0.009)$, (B) IL-6 and vagal tonus ( $r=-0.7, P<0.0002),(\mathbf{C})$ TNF- $\alpha$ and GSH/GSSG ( $r=-0.60, P<0.003),(\mathbf{D})$ IL-6 and MPI ( $r=0.65$, $P<0.0002)$, (E) sympathetic tonus and MPI $(r=0.55, P<0.006)$, and $(\mathbf{F}) \mathrm{MPI}$ and physical capacity $(r=-0.55, P<0.003)$.

Abbreviations: GSH/GSSG, glutathione redox balance; IL, interleukin; MPI, myocardial performance index; OC, old (24 months old) control; OO, old ovariectomized; TNF, tumor necrosis factor; YC, young (5 months old) control; YO, young ovariectomized.

among all groups. This finding may be associated with the fact that with the gradual decline of estrogen in the climacteric period, low-density lipoprotein receptor activity is decreased. ${ }^{27}$

\section{Autonomic and cardiac function}

In the study on the influence of the autonomic nervous system in the inflammatory response, Satapathy et $\mathrm{a}^{28}$ found a suppression of circulating TNF- $\alpha$ levels in rats treated with galantamine (cholinesterase inhibitor), together with a reduction in body weight, abdominal adiposity, and insulin resistance. In this sense, the following events could be shown in our trial: dysautonomia, increased inflammatory markers, increased body and WAT weight, and insulin resistance in all groups when compared with YC. The systolic function was similar among the four groups. However, the evaluation of diastolic function showed decreased E/A ratio, while isovolumetric relaxation time and deceleration $\mathrm{E}$ was increased in old (OC and $\mathrm{OO}$ ) when compared with young groups (YC and YO). Abnormalities in these parameters suggest that the left ventricle cannot fill properly in the period between contractions. Left ventricular morphometric evaluations indicated that old rats (OC and $\mathrm{OO}$ ) presented a higher left ventricular mass and increased left ventricular diameter during diastole when compared with young groups ( $\mathrm{YC}$ and $\mathrm{YO}$ ). Importantly, the global index of heart function quantified by the 
MPI was higher in old when compared with young animals, thus suggesting that the aging process may contribute to this impairment. Furthermore, a negative correlation has been observed between physical capacity and MPI ( $r=-0.55)$. In fact, physical capacity was decreased in aged animals (OC and $\mathrm{OO}$ ) throughout the protocol when compared with young ones (YC and YO). Additionally, ovariectomy (YO and OO) induced lower physical capacity in young or old rats when compared with the intact controls, at the end of the protocol. The worsening performance observed in aged ovariectomized rats (OO group) suggests that deprivation of ovarian hormones in aging animals may lead to additional reduction in both lean mass (sarcopenia) and bone mass (osteopenia). ${ }^{22}$

It is important to emphasize that left ventricular diastolic dysfunction and morphometric alterations can result from several factors, such as increased collagen concentration and fibrosis and decreased compliance, which is influenced by the autonomic nervous system and the renin-angiotensin system. $^{29,30}$

Indeed, increased sympathetic activity to the heart was observed in aged and ovariectomized animals regardless of age, indicating that this could be one of the mechanisms underlying diastolic function impairment in old rats. In addition, the reduction in parasympathetic function observed only in aged animals may lend further support to the finding of a worsened heart function in these animals. These autonomic changes were accompanied by changes in baseline HR when comparing $\mathrm{YC}$ and $\mathrm{YO}$ with $\mathrm{OC}$ and $\mathrm{OO}$ groups, possibly caused by disorders observed in cardiac autonomic balance. ${ }^{31}$ Indeed, it has been shown in this trial that cardiac vagal tonus decreased in old animals (OC and OO), whereas sympathetic tonus increased. However, old groups showed an IHR reduction that may be due to the age-related changes in function of the sinus node. ${ }^{32,33}$ The findings in this study suggest that ovariectomy aggravates autonomic dysfunction observed in older animals. This increase is probably due to a reduction in the vagal effect, which may be causing a reduction of the reflex bradycardia response mediated by baroreceptors. Although aging produces a decline in the production of ovarian hormones, the removal of the ovaries in older females abolishes the production of these hormones. ${ }^{34,35}$ And these changes may have led to a greater autonomic dysfunction in the OO group. Indeed, the comparison between the young groups ( $\mathrm{YC}$ and $\mathrm{YO}$ ) has shown that the aging process changes cardiac and autonomic profile in female rats. In addition, young female ovariectomized are similar to intact elderly rats, suggesting that many of the differences observed in young groups ( $\mathrm{YC}$ and $\mathrm{YO}$ ) have not been observed in the old groups ( $\mathrm{OC}$ and $\mathrm{OO}$ ) and these changes are probably due to the aging process. Regarding MAP, an increase in YO and $\mathrm{OO}$ rats was observed in comparison with their intact controls. Increased inflammatory status in ovariectomized rats associated with the increased oxidative stress in these groups might be associated with nitric oxide reduction and, consequently, with the changes in AP. ${ }^{33}$

The underlying mechanisms mediating abnormal vascular function with aging and menopause remain controversial, but in vitro $17 \beta$-estradiol treatment increases the elastin and decreases collagen production in the vascular smooth muscle. Besides, 17 $\beta$-estradiol also exerts protective effects in the prevention of cardiomyocyte apoptosis. Further work needs to be done for a better understanding of this kind of supplementation, since myocardial aging is associated with a myriad of changes, including left ventricular hypertrophy, heart failure, and increased myocardial stiffness that can be correlated with inflammation and oxidative stress generation.

\section{Inflammation and oxidative stress}

The decline of immune activation after aging remains unclear despite that there are several trials focusing on it. Inflammatory processes combined with cytokine release are important steps in building a response to tissue injury and they play an active role in cardiac function. Thus, both OC and $\mathrm{OO}$ groups were found to have increased IL-6 levels when compared with YC and YO, whereas TNF- $\alpha$ was higher in old groups when compared with YC. Following the hypothesis of a cholinergic anti-inflammatory pathway via vagus nerves, these results would be associated with the lower parasympathetic participation observed in old groups. ${ }^{36,37}$ In this sense, the correlations found between IL-6 and vagal tonus ( $r=-0.7)$ and sympathetic tonus ( $r=0.65)$ would further reinforce this hypothesis (Figure 1).

Indeed, oxidative stress as expressed by TBARS measurements was enhanced in both $\mathrm{YO}$ and $\mathrm{OO}$ groups in relation to $\mathrm{YC}$ and $\mathrm{OC}$ groups, indicating that the impairment of heart function in ovariectomized animals may be related to increased oxidative stress in the tissue. It is well established that the increase in lipid membrane peroxidation may be explained by sequestration of hydrogen polyunsaturated fatty acid located in the cell membrane. This, in turn, leads to the loss of selectivity of iron exchange and release of organelles content, together with the formation of toxic substances causing cell death. ${ }^{38}$ Indeed, a previous study demonstrated a positive correlation between AP and lipid peroxidation, thus reinforcing the role of oxidative stress in AP changes during hormone deprivation. ${ }^{5}$ The decreased 
lipoperoxidation observed in both YC and OC groups when compared with ovariectomized rats seems to be related to the antioxidant properties of estrogens and their regulatory action on antioxidant enzymes. ${ }^{30,39}$

Moreover, protein oxidation was higher in $\mathrm{OO}$ when compared with YC group indicating that not only the advancing age, but also the ovariectomy increased protein damage. ${ }^{40,41}$

OVX has caused an increase in GPX activity in YO and OO, when compared with YC and OC. These changes could be explained as compensatory mechanisms since CAT was not different among groups and both enzymes compete for the same substrate (hydrogen peroxide) ${ }^{42}$ It should be noted that the GSH/GSSG decreased in YO, OC, and OO, when compared with $\mathrm{YC}$. Moreover, $\mathrm{YO}$ and $\mathrm{OO}$ had a further decrease in GSH/GSSG when compared with OC group. These results indicate that aging and/or OVX might be associated with impairment in redox balance. In this aspect, the data obtained by correlation analyses reinforce the hypothesis that cardiac remodeling is associated with increased oxidative stress ${ }^{43}$ and inflammation (correlations between TNF- $\alpha$ with GSH/ GSSG, $r=-0.6$; MPI with IL-6 and TNF- $\alpha, r=0.65$ and $r=0.5$, respectively). Consequently, both mechanisms may lead to the impairment of global left ventricular function.

\section{Study limitation}

Some limitations in the current study should be pointed out, such as no data on mortality. In addition, estrogen- and progesterone-related data were not quantified in this protocol, although we have previously performed this evaluation.

\section{Clinic implications}

In women, aging coincides with menopause, during which there is a significant reduction in the production of ovarian hormones. Information in this study has shown that aging alone induces autonomic, biochemical, and functional changes that can be exacerbated by menopause, thus, giving support to the search for early interventions during this physiological process that can mitigate the deleterious effects of aging in female individuals.

\section{Conclusion}

It was shown in this study that OVX aggravates the impairment of cardiac and functional effects of aging in female rats, probably associated with exacerbated autonomic dysfunction, inflammation, and oxidative stress. In this sense, preventing and/or attenuating autonomic dysfunction triggered by aging process may result in a lower proinflammatory and oxidative stress profile, along with preserved cardiac function, possibly leading to improved quality of life and life expectancy of this female population after OVX.

\section{Acknowledgment}

This study was supported by CNPq (563961/2010- MCI, KA; 484713/2011- MCI, KA; 306 011/2010-MCI; 457200/2014-6; 309292/2014-0 KA); CAPES (PVE-074/ 12-JFM, KA, MM, MCI; 88881.062178/2014-01 KA), and FAPESP 2012/20141-5 KA.

\section{Disclosure}

The authors report no conflicts of interest in this work.

\section{References}

1. Govindaraju DR, Pencina KM, Raj DS, Massaro JM, Carnes BA, D'Agostino RB. A systems analysis of age-related changes in some cardiac aging traits. Biogerontology. 2014;15(2):139-152.

2. World Health Organization. Cardiovascular Diseases (CVDs). 2015; Fact Sheet $\mathrm{N}^{\circ}$ 317: Available from: www.who.int/mediacentre/ factsheets/fs317/en/. Accessed February 21, 2015.

3. American Heart Association's Go Red For Women and Ad Council Launch National Public Service Advertising Campaign to Address a Leading Killer of Women in the U.S. Heart Disease. EINNews, November 11, 2014

4. Kochanek KD, Xu J, Murphy SL, Minino AM, Kung HC. Deaths: final data for 2009. Natl Vital Stat Rep. 2011;60(3):1-116.

5. Irigoyen MC, Paulini J, Flores LJ, et al. Exercise training improves baroreflex sensitivity associated with oxidative stress reduction in ovariectomized rats. Hypertension. 2005;46(4):998-1003.

6. Flores LJ, Figueroa D, Sanches IC, et al. Effects of exercise training on autonomic dysfunction management in an experimental model of menopause and myocardial infarction. Menopause. 2010;17(4):712-717.

7. Marcondes FK, Bianchi FJ, Tanno AP. Determination of the estrous cycle phases of rats: some helpful considerations. Braz J Biol. 2002; 62(4A):609-614.

8. Latour MG, Shinoda M, Lavoie JM. Metabolic effects of physical training in ovariectomized and hyperestrogenic rats. J Appl Physiol (1985). 2001;90(1):235-241.

9. Sanches IC, de Oliveira Brito J, Candido GO, et al. Cardiometabolic benefits of exercise training in an experimental model of metabolic syndrome and menopause. Menopause. 2012;19(5):562-568.

10. Flues K, Paulini J, Brito S, et al. Exercise training associated with estrogen therapy induced cardiovascular benefits after ovarian hormones deprivation. Maturitas. 2010;65(3):267-271.

11. Brooks GA, White TP. Determination of metabolic and heart rate responses of rats to treadmill exercise. J Appl Physiol Respir Environ Exercise Physiol. 1978;45(6):1009-1015.

12. Bonora E, Moghetti $\mathrm{P}$, Zancanaro C, et al. Estimates of in vivo insulin action in man: comparison of insulin tolerance tests with euglycemic and hyperglycemic glucose clamp studies. J Clin Endocrinol Metab. 1989;68(2):374-378.

13. Wichi R, Malfitano C, Rosa $\mathrm{K}$, et al. Noninvasive and invasive evaluation of cardiac dysfunction in experimental diabetes in rodents Cardiovasc Diabetol. 2007;6:14.

14. Moraes-Silva IC, De La Fuente RN, Mostarda C, et al. Baroreflex deficit blunts exercise training-induced cardiovascular and autonomic adaptations in hypertensive rats. Clin Exp Pharmacol Physiol. 2010; 37(3):e114-e120.

15. Negrao CE, Moreira ED, Brum PC, Denadai ML, Krieger EM. Vaga and sympathetic control of heart rate during exercise by sedentary and exercise-trained rats. Braz J Med Biol Res. 1992;25(10):1045-1052. 
16. Buege JA, Aust SD. Microsomal lipid peroxidation. Methods Enzymol. 1978;52:302-310.

17. Reznick AZ, Packer L. Oxidative damage to proteins: spectrophotometric method for carbonyl assay. Methods Enzymol. 1994;233:357-363.

18. Marklund SL. Superoxide dismutase isoenzymes in tissues and plasma from New Zealand black mice, nude mice and normal BALB/c mice. Mutat Res. 1985;148(1-2):129-134.

19. Aebi H. Catalase in vitro. Methods Enzymol. 1984;105:121-126.

20. Flohe L, Gunzler WA. Assays of glutathione peroxidase. Methods Enzymol. 1984;105:114-121.

21. Anderson ME. Determination of glutathione and glutathione disulfide in biological samples. Methods Enzymol. 1985;113:548-555.

22. Ferreira JA, Foley AM, Brown M. Sex hormones differentially influence voluntary running activity, food intake and body weight in aging female and male rats. Eur J Appl Physiol. 2012;112(8):3007-3018.

23. Shimomura K, Shimizu H, Tsuchiya T, Abe Y, Uehara Y, Mori M. Is leptin a key factor which develops obesity by ovariectomy? Endocr J. 2002;49(4):417-423.

24. Landsberg L, Aronne LJ, Beilin LJ, et al. Obesity-related hypertension: pathogenesis, cardiovascular risk, and treatment: a position paper of The Obesity Society and the American Society of Hypertension. J Clin Hypertens (Greenwich). 2013;15(1):14-33.

25. Gulcelik NE, Halil M, Ariogul S, Usman A. Adipocytokines and aging: adiponectin and leptin. Minerva Endocrinol. 2013;38(2):203-210.

26. Wronska A, Lawniczak A, Wierzbicki PM, Goyke E, Sledzinski T, Kmiec Z. White adipose tissue depot-specific activity of lipogenic enzymes in response to fasting and refeeding in young and old rats. Gerontology. 2015;61:448-455.

27. Kuller LH, Meilahn EN, Cauley JA, Gutai JP, Matthews KA. Epidemiologic studies of menopause: changes in risk factors and disease. Exp Gerontol. 1994;29(3-4):495-509.

28. Satapathy SK, Ochani M, Dancho M, et al. Galantamine alleviates inflammation and other obesity-associated complications in high-fat diet-fed mice. Mol Med. 2011;17(7-8):599-606.

29. Mostarda C, Moraes-Silva IC, Salemi VM, et al. Exercise training prevents diastolic dysfunction induced by metabolic syndrome in rats. Clinics (Sao Paulo). 2012;67(7):815-820.

30. Arnal JF, Clamens S, Pechet C, et al. Ethinylestradiol does not enhance the expression of nitric oxide synthase in bovine endothelial cells but increases the release of bioactive nitric oxide by inhibiting superoxide anion production. Proc Natl Acad Sci US A. 1996;93(9):4108-4113.
31. Tezini GC, Becari C, Zanotto CZ, Salgado MC, Passaglia Rde C, Souza HC. Ageing is the main determinant of haemodynamics and autonomic cardiac changes observed in post-menopausal female rats. Auton Neurosci. 2013;174(1-2):36-41.

32. Joyner MJ. Not so fast: intrinsic heart rate vs. beta-adrenergic responsiveness in the aging human heart. J Appl Physiol (1985). 2008;105(1): 3-4.

33. Rubio-Ruiz ME, Perez-Torres I, Soto ME, Pastelin G, Guarner-Lans V. Aging in blood vessels. Medicinal agents FOR systemic arterial hypertension in the elderly. Ageing Res Rev. 2014;18:132-147.

34. Lopes GS, Ferreira AT, Oshiro ME, et al. Aging-related changes of intracellular $\mathrm{Ca} 2+$ stores and contractile response of intestinal smooth muscle. Exp Gerontol. 2006;41(1):55-62.

35. Lopes GS, Mora OA, Cerri P, et al. Mitochondrial alterations and apoptosis in smooth muscle from aged rats. Biochim Biophys Acta. 2004; 1658(3):187-194.

36. Andersson U, Tracey KJ. Neural reflexes in inflammation and immunity. J Exp Med. 2012;209(6):1057-1068.

37. Borovikova LV, Ivanova S, Zhang M, et al. Vagus nerve stimulation attenuates the systemic inflammatory response to endotoxin. Nature. 2000;405(6785):458-462.

38. Hershko C. Mechanism of iron toxicity and its possible role in red cell membrane damage. Semin Hematol. 1989;26(4):277-285.

39. Barbacanne MA, Rami J, Michel JB, et al. Estradiol increases rat aorta endothelium-derived relaxing factor (EDRF) activity without changes in endothelial NO synthase gene expression: possible role of decreased endothelium-derived superoxide anion production. Cardiovasc Res. 1999;41(3):672-681.

40. Carrard G, Dieu M, Raes M, Toussaint O, Friguet B. Impact of ageing on proteasome structure and function in human lymphocytes. Int J Biochem Cell Biol. 2003;35(5):728-739.

41. Herrmann J, Soares SM, Lerman LO, Lerman A. Potential role of the ubiquitin-proteasome system in atherosclerosis: aspects of a protein quality disease. J Am Coll Cardiol. 2008;51(21):2003-2010.

42. De Angelis KL, Cestari IA, Barp J, et al. Oxidative stress in the latissimus dorsi muscle of diabetic rats. Braz J Med Biol Res. 2000;33(11): 1363-1368.

43. Rosa CM, Xavier NP, Henrique Campos D, et al. Diabetes mellitus activates fetal gene program and intensifies cardiac remodeling and oxidative stress in aged spontaneously hypertensive rats. Cardiovasc Diabetol. 2013;12:152
Clinical Interventions in Aging

\section{Publish your work in this journal}

Clinical Interventions in Aging is an international, peer-reviewed journal focusing on evidence-based reports on the value or lack thereof of treatments intended to prevent or delay the onset of maladaptive correlates of aging in human beings. This journal is indexed on PubMed Central, MedLine,

\section{Dovepress}

CAS, Scopus and the Elsevier Bibliographic databases. The manuscript management system is completely online and includes a very quick and fair peer-review system, which is all easy to use. Visit http://www.dovepress. com/testimonials.php to read real quotes from published authors. 